\title{
Extraction Techniques in Sample Preparation
}

Created by: Victoria Samanidou

Sample preparation is the most crucial step in the analytical procedure designed for implementation in any analytical application (food analysis, bionalysis, forensics, toxicology, environmental monitoring etc). It is the limiting factor in chemical analysis since it is time consuming and it can potentially introduce errors. No one can doubt that the best approach would be the direct introduction of the sample to the instrument, however this is rarely feasible. Efficient sample pretreatment is inevitably required as the instrument technology has produced highly sophisticated and sensitive analytical equipment.

Hence, the analytical scientists have to develop and apply a suitable sample preparation protocol that ensures that the composition of the sample remains unchanged, no impurities are introduced during handling, all interferences have been left back, the analytes' concentration is not only at detectable levels, but it can also be quantified precisely and accurately and that the matrix of the sample is compatible with the analytical technique.

Extraction techniques are the most powerful tool in hands of the analytical chemists and lab practitioners. Either sorbent based or solvent based, extraction techniques provide the necessary tool that can be used to handle the sample in a way that all information in it can be revealed, all advantages in instrumentation have been exploited to the fullest and the lifetime of the instrument is prolonged in a seamless operation mode.

\section{Extraction techniques in Sample Preparation}

\section{Victoria F. Samanidou}

Laboratory of Analytical Chemistry, Department of Chemistry,

Aristotle University of Thessaloniki, 54124 Thessaloniki, Greece

Corresponding author: Victoria Samanidou. Tel:+30231997698

FAX: +302310997719, e-mail:samanidu@chem.auth.gr

\section{Introduction}

Sample preparation is the most crucial step in the analytical procedure designed for implementation in any analytical application (food analysis, bionalysis, forensics, toxicology, environmental monitoring etc) (Figure 1). It is the limiting factor in chemical analysis since it is time consuming and it can potentially introduce errors. No one can doubt that the best approach would be the direct introduction of the sample to the instrument, however this is feasible only in limited applications. Efficient sample pretreatment is inevitably required as the instrument technology has produced highly sophisticated and sensitive analytical equipment. 


\section{General Steps in Sample Analysis}

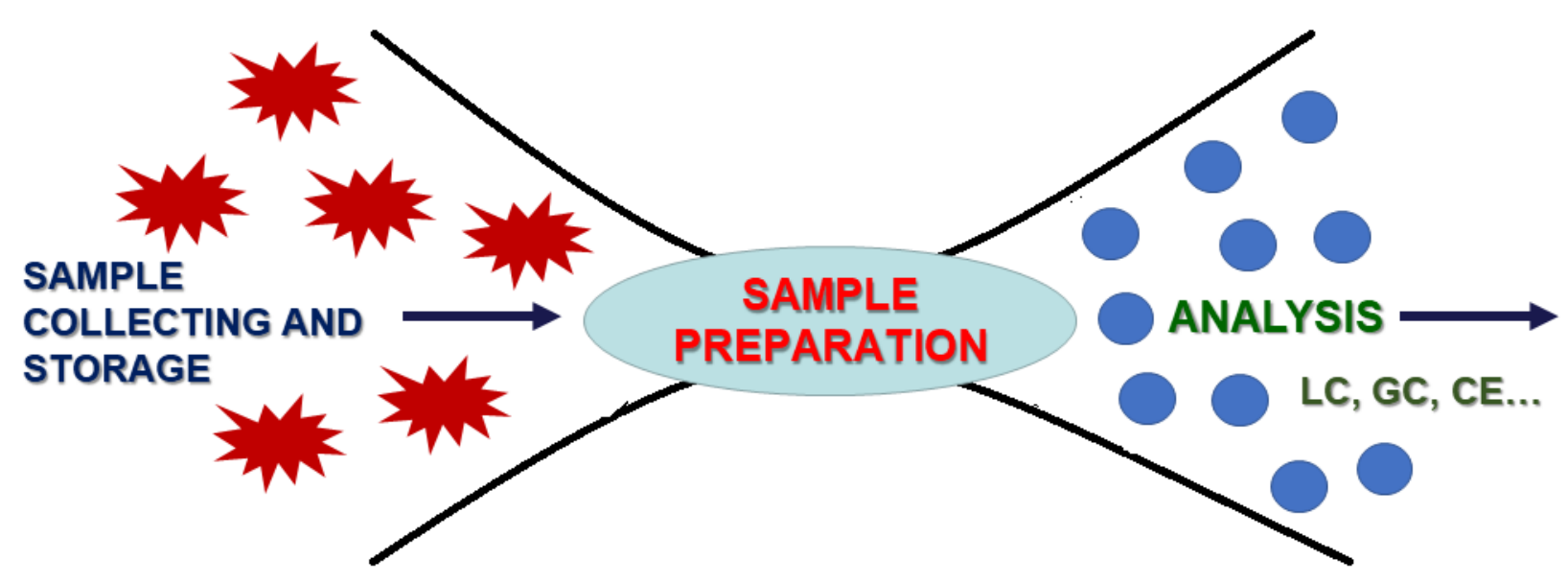

Figure 1. General steps in sample analysis.

Hence, the analytical scientists have to develop and apply a suitable sample preparation protocol that ensures that the composition of the sample remains unchanged, no impurities are introduced during handling, all interferences have been left back, the analytes' concentration is not only at detectable levels but it can also be quantified precisely and accurately, and that the matrix of the sample is compatible with the analytical technique (Figure 2).

\section{Objectives of Extraction Techniques in Sample Preparation}

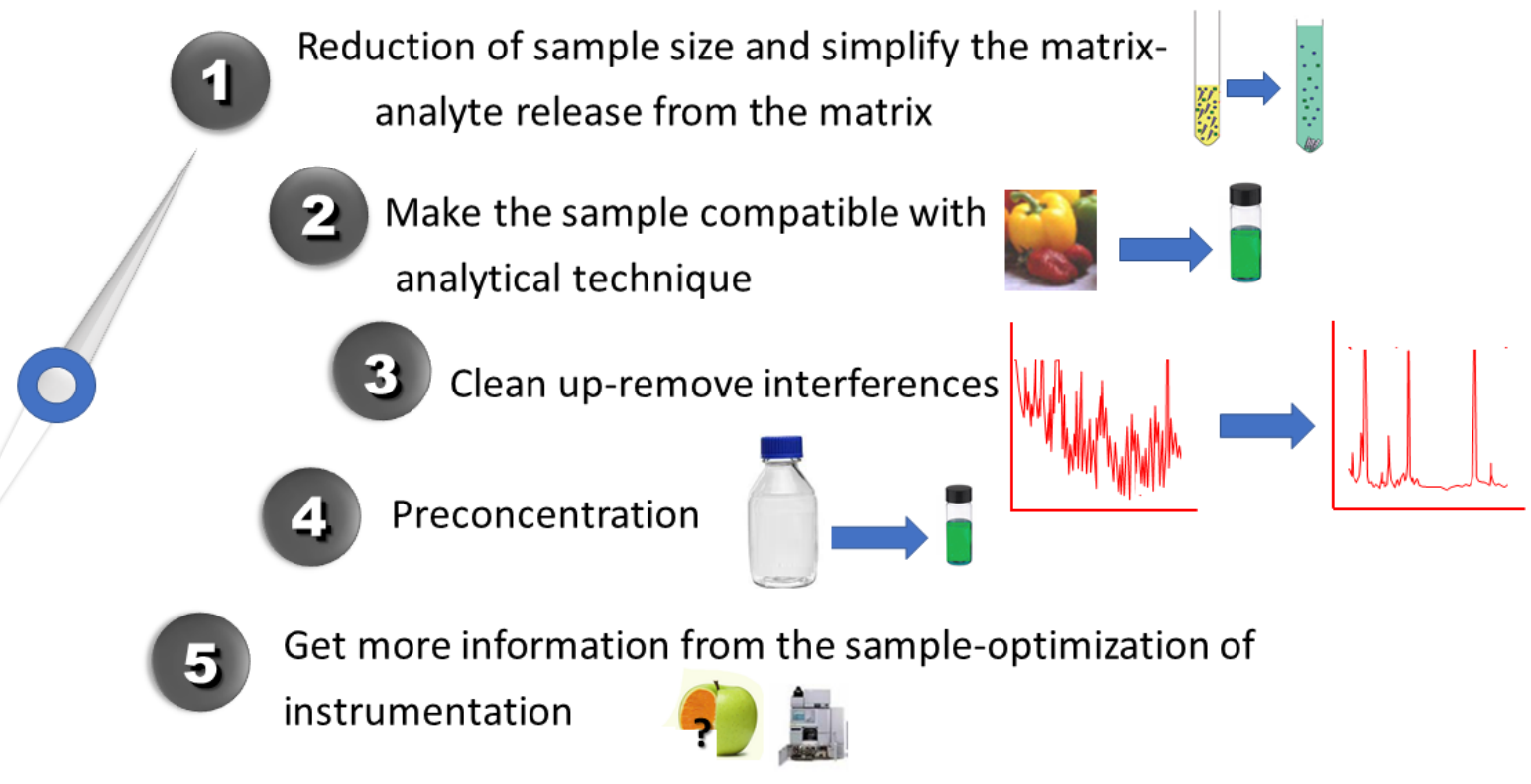

Figure 2. Objectives of Sample Preparation.

In order to achieve all the above the analytical chemist must apply several sub- steps, such as dilution, filtration, deproteinization, centrifugation, purification, derivatization, analyte isolation, clean-up/interference removal, preconcentration, evaporation etc. Among all these steps, beyond any doubt, extraction techniques are the most powerful tool in hands of the analyst. A tool that can be used to handle the sample in a way that all information in it can be revealed, all advantages in instrumentation have been exploited to the fullest and the lifetime of the instrument is prolonged in a seamless operation mode. 
Extraction can be either sorbent- based or solvent- based and is being applied in many configurations throughout all years, in numerous analyses.

There are many choices to fit the purpose of any analytical problem. Physicochemical characteristics of target compounds, sample matrix and available instrumentation are decisive for the choice of best extraction technique as well as to find the optimum extraction conditions. To this end many different solvents, packing materials, different mechanisms and design configurations are involved.

Solvent, temperature and pressure-based techniques are basically applied but these can be assisted by vortex, microwaves, sonication, to ensure analytes stability and high recovery rates.

Selective sorbents designed for specific analytes (immunosorbents, molecularly imprinted polymers (MIPs), mixed mode sorbents are providing the desired selectivity. Moreover, novel advanced materials favored by the evolution of nanotechnology with higher surface area and sorption capacities improved the efficiency of traditional materials.

Extraction techniques can be classified as follows:

- Flow through Equilibrium \& pre-equilibrium

- Batch Equilibrium \& pre-equilibrium

- Steady state Exhaustive \& Non-exhaustive

Examples of each category are shown in Figure 3.

\section{Classification of Extraction techniques}

\begin{tabular}{|c|c|c|c|c|}
\hline \multicolumn{2}{|c|}{$\begin{array}{l}\text { Flow through Equilibrium \& pre- } \\
\text { equilibrium }\end{array}$} & \multicolumn{2}{|c|}{$\begin{array}{l}\text { Batch Equilibrium } \\
\text { \& pre-equilibrium }\end{array}$} & \multirow{3}{*}{\begin{tabular}{|l|}
$\begin{array}{l}\text { Steady state } \\
\text { Exhaustive \& Non- } \\
\text { exhaustive }\end{array}$ \\
\begin{tabular}{|l} 
Membrane \\
Dialysis \\
Microdialysis
\end{tabular}
\end{tabular}} \\
\hline Exhaustive & Non-exhaustive & Exhaustive & Non-exhaustive & \\
\hline $\begin{array}{l}\text { Purge \& Trap } \\
\text { Sorbent Trap } \\
\text { SPE } \\
\text { SFE }\end{array}$ & $\begin{array}{l}\text { In tube } \\
\text { SPME }\end{array}$ & $\begin{array}{l}\text { LLE } \\
\text { Soxhlet } \\
\text { Sorbents } \\
\text { FPSE }\end{array}$ & $\begin{array}{l}\text { Headspace } \\
\text { LLME } \\
\text { SPME }\end{array}$ & \\
\hline
\end{tabular}

Figure 3. Classification of extaction techniques

In earlier times Soxhlet extraction, liquid- liquid extraction (LLE) and solid- liquid extraction (SLE) were the prevailing techniques in analytical methodologies. However, the situation has changed, and many extraction modes have been introduced by researchers all over the world. [1][2][3][4][5][6][7][8][9][10][11][12][13][14]

High throughout, sensitivity, rapidity and ease of use, along with high recovery rates are the prerequisites in new developments. Additionally, modern extraction techniques must comply with the principles of sustainable development and green chemistry demands in order to ensure operator and public safety and environmental preservation. 


\section{Encyclopedia}

To this direction micro-extraction configurations have replaced traditional extraction modes, in order to reduce the energy consumption, reagents consumption and waste generation.

Less toxic media are available to replace toxic solvents. Ionic liquids, deep eutectic solvents, superheated water, supercritical carbon dioxide are among the most representative media.

Miniaturized techniques are promising to facilitate the sample handling step and to increase efficiency and accuracy of the method, using low quantities of sorbent materials and requiring no solvent or low solvent volumes together with low sample volumes. Selectivity, sensitivity and lower quantification limits are among the impressive performance characteristics of these techniques which are required to meet the legislation criteria.

The list of extraction techniques that follows is only indicative and has obviously no end, because every day in the literature we can see new or modified ones:

- Liquid-Liquid Extraction (LLE)

- Solid Phase Extraction (SPE)

- Magnetic solid phase extraction (MSPE)

- Micro- Solid Phase Extraction ( $\mu \mathrm{SPE}$ )

- dispersive solid phase extraction (dSPE)

- Molecularily imprinted Solid Phase Extraction

- matrix solid phase dispersion (MSPD)

- disposable pipette extraction (DPX)

- Solid phase Microextraction (SPME)

- Headspace-SPME

- In-tube SPME

- Microextraction in Packed Syringe (MEPS)

- Liquid phase microextraction (LPME)

- Two phase and three phase LPME

- Single drop microextraction (SDME)

- dispersive liquid liquid microextraction (DLLME)

- Hollow fiber supported Liquid phase microextraction (HFLPME)

- Gas phase extraction

- Supercritical fluid extraction (SFE)

- Accelerated solvent extraction (ASE), or Pressurized liquid extraction (PLE)

- Fabric phase sorptive extraction (FPSE)

- Capsule Phase Microextrcation (CPME)

- Stir bar sorptive extraction (SBSE)

- Stir cake sorptive extraction

- Rotating-disc sorbent extraction (RDSE)

- Quick, Easy, Cheap, Effective, Rugged and Safe extraction (QuEChERS)

- Membrane based extraction

- Electromembrane extraction

- Cloud point extraction

- Soxhlet extraction

- Microwave assisted solvent extraction (MASE)

- Vortex assisted extraction

- Ultrasound assisted extraction

- Paper based sorptive extraction

- Supported liquid extraction.

Although the best sample preparation would be no sample preparation, the second-best choice is "just enough" sufficient sample preparation, which would provide selective, fast, cheap extraction in terms also of the twelve principles of green chemistry. And this is achieved by the implementation of a suitable extraction technique. 


\section{Conclusions}

Current trends in analytical chemistry, focus on miniaturization, simplification, and automation, to deal with high throughput in routine analysis, also following the concept of green analytical chemistry.

Sample preparation is and will always be the most vital step in chemical analysis. Numerous techniques, methods, methodologies, configurations and approaches are published in literature offering a wide range of analytical tools to the lab practitioner who has to deal with various complex sample matrices

Extraction and green microextraction techniques are applied to guarantee the best analytical performance. The future perspectives for microextraction techniques depend on the incorporation of even less toxic solvents, the development of more powerful sorbent materials, further miniaturization, full automation and on-line coupling capability with analytical instruments.

Suggested further reading is provided in References section.

\section{References}

1. A. Kabir A, KG. Furton, A. Malik. Innovations in sol-gel microextraction phases for solvent-free sample preparation in analytical chemistry. Trends in Analytical Chemistry. 45, 2013, 197-218.

2. S. Armenta, S. Garrigues, M. de la Guardia. Green Extraction Techniques: The role of green extraction techniques in Green Analytical Chemistry. Trends in Analytical Chemistry, Volume 71, 2015, Pages 2-8.

3. V. Samanidou, M. Kissoudi. Recent advances in applications of ionic liquids in miniaturized microextraction techniques, Molecules, 2018, 23(6), 1437

4. E. Zilfidou, A.Kabir, K. G. Furton. V Samanidou. Fabric Phase Sorptive Extraction: Current State of the Art and Future Perspectives, Separations, 2018, 5(3), 40;

5. N. Manousi, G. Zachariadis, E. Deliyanni, V. Samanidou. Applications of Metal-Organic Frameworks in Food Sample Preparation. Molecules, 2018, 23(11), 2896.

6. L.Ramos, Use of new tailored and engineered materials for matrix solid-phase dispersion, Trends in Analytical Chemistry, Volume 118, 2019, Pages 751-758.

7. M.Lashgari, V.Singh, J. Pawliszyn. A critical review on regulatory sample preparation methods: Validating solid-phase microextraction techniques (Review). Trends in Analytical Chemistry. Volume 119, 2019, Article number 115618

8. F. A.Hansen, S. Pedersen-Bjergaard, Emerging Extraction Strategies in Analytical Chemistry. Analytical Chemistry, 92 (1), $2020,2-$ 15.

9. E.Psillakis, Vortex-assisted liquid-liquid microextraction revisited, Trends in Analytical Chemistry 113, 2019, pages 332-339.

10. N.Kalogiouri, V.Samanidou. Recent Trends in the Development of Green Microextraction Techniques for the Determination of Hazardous Organic Compounds in Wine. Current Analytical Chemistry 2019, Vol. 15, No. 7, 788-800, 2019.

11. M. Abdel-Rehim, S. Pedersen-Bjergaard, A. Abdel-Rehim, S, Cárdenas, R. Lucena, M. Mahdi Moein, M. Miró. Microextraction approaches for bioanalytical applications: An overview. Journal of Chromatography A, 1616, 2020, 460790

12. A. Tartaglia, M. Locatelli, V.Samanidou. Trends in the Analysis of Biopharmaceuticals by HPLC. Current Analytical Chemistry, 2020, $16,52-58$.

13. V. Alampanos, V. Samanidou, I. Papadoyannis Trends in sample preparation for the HPLC determination of penicillins in bio-Fluids. J. of Applied Bioanalysis (2019) Vol. 5, No. 1, 9-17.

14. N. Manousi, E. Rosenberg, E. Deliyanni, G. Zachariadis, V. Samanidou. Magnetic solid-phase extraction of organic compounds based on graphene oxide nanocomposites. Molecules 2020, 25(5), 1148.

\section{Keywords}

extraction; microextraction; sample preparation; sorptive extraction; liquid extraction 
